\title{
American Journal of Engineering Research and Reviews
} (ISSN:2637-3785)

\section{Design of "S" type track carbon free car based on cam mechanism}

\author{
Xing $\mathrm{Li}^{{ }^{*}}$, Zhizhe Wang ${ }^{1}$, Dachao Yang ${ }^{1}$, Jiangping Tang ${ }^{1}$, Tianlong Wang ${ }^{2}$ \\ ${ }^{1}$ College of Mechanical \& Power Engineering, China Three Gorges University, Yichang, 443002, \\ China. \\ ${ }^{2}$ College of Civil Engineering \& Architecture, China Three Gorges University, Yichang, 443002, \\ China.
}

\begin{abstract}
Reverse part model based on motion trajectory is a common method in mechanism design. Compared with the traditional Xing Li calculation method, it is simple and practical, and can simulate the reconstruction of the trajectory. In this paper, S-type carbonfree car was taken as an example. Firstly, the motion trajectory of the ideal car path was given. UG software was used to obtain the curve characteristics, established a mathematical model, and took the curve into the model to obtain the data of cam coordinate points. Then the MATLAB software was used to draw the cam contour and analyzed the feasibility of cam operation. The vehicle trajectory simulation was realized in MATLAB environment. Finally, the reasonable cam contour coordinate point data was imported into UG software to establish a 3D model of the cam, and the car track was repeatedly optimized to get the optimal cam shape. The rationality and the accuracy of the model were verified by the carbon-free car made by the scheme, and the corresponding guidance was provided for the research of others.
\end{abstract}

Keywords: Cam; MATLAB software; UG software; Carbonless car
${ }^{*}$ Correspondence to Author:

College of Mechanical \& Power Engineering, China Three Gorges University, Yichang, 443002, China. How to cite this article:

Xing Li, Zhizhe Wang, Dachao Yang, Jiangping Tang, Tianlong Wang. Design of "S" type track carbon free car based on cam mechanism. American Journal of Engineering Rsearch and Reviews, $2021 ; 4: 27$

\section{eScî̀Pub}

eSciPub LLC, Houston, TX USA. Website: http://escipub.com/ 


\section{Introduction}

Carbon-free car is a kind of green car with energy saving and environmental protection. The kinetic energy in the moving process is provided by the potential energy generated in the falling process of weights. The car is driven forward by gears and other transmission mechanisms, and periodic self-steering is achieved through special mechanical structure in the moving process. Chen et al. [1] realized the double "8" character walking law of the car through the incomplete gear mechanism; Ai et al. ${ }^{[2]}$ used Simulink to obtain the optimal driving parameters of carbon-free car around obstacles through simulation analysis, which could maxi- mize the repetition of trajectory detour. Based on the concept of energy saving and green environmental protection, Liu et al. ${ }^{[3]}$ designed a kind of conversion from gravitational potential energy to kinetic energy, and its energy replacement approach was shown in Fig. 1.Wang et al. ${ }^{[4]}$ using disc groove cam as car steering mechanism, was proposed based on the cam control carbon-free car design method of automatic steering, and put forward the path reverse method: according to the proposed track reversed cam contour coordinates information, finally it was concluded that the ideal cam profile, the method of carbon-free car its rationality was verified in practice.

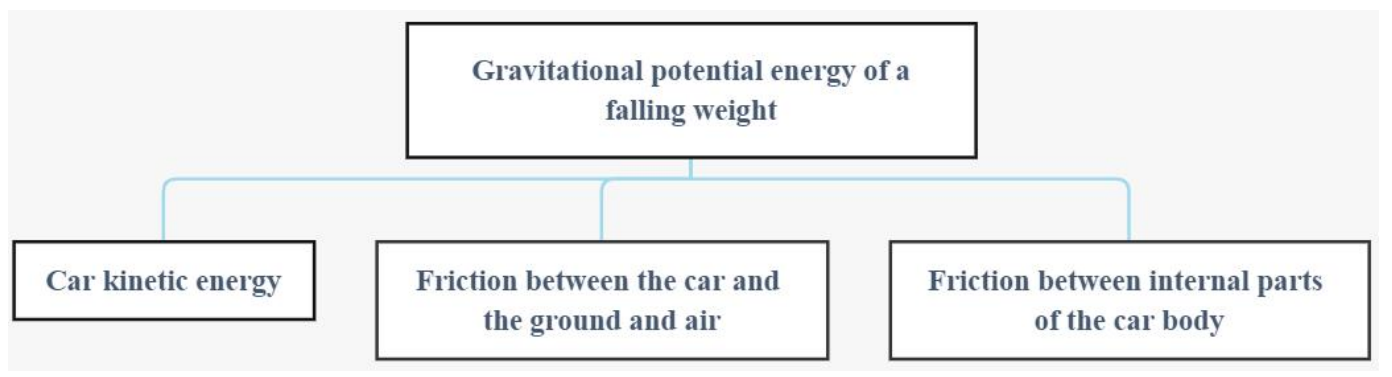

Fig. 1 Vehicle energy conversion path

\section{Design scheme}

\subsection{Overall structure}

The trolley adopts a three-wheel structure, in which the front wheel is the steering wheel and the two rear wheels are the traveling wheel. Fig. 2 is a schematic diagram of a carbon-free car. We used the rear wheel dual drive mode, at the same time considered when the car turned, the speed of the two rear wheels was different. To achieve differential, we used one-way bearings between the rear axle drive gear and the rear axle.

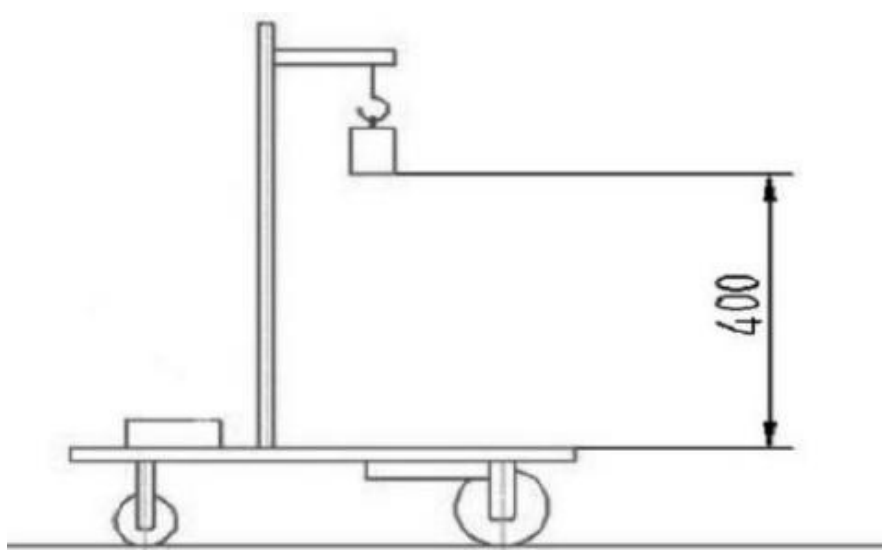

Fig. 2 Schematic diagram of carbon-free car 


\subsection{Prime mover structure}

The kinetic energy of the car is provided by the weight, one end of the winding relates to the car driving shaft. one end of the hanging weight, winding through the fixed pulley at the top of the column. when the weight falls, driving the driving shaft to rotate, the driving shaft to drive the driving gear, and then drive the rear wheel shaft gear to rotate, in order to achieve the rear wheel drive. In order to avoid the situation that the car can't start because the driving torque at the start moment was less than the friction torque between the car and the ground, we installed a starter on the driving shaft, which could provide a larger torque at the start moment.

\subsection{Transmission mechanism}

The transmission mechanism should meet the requirements of smooth transmission and high transmission efficiency. Our design used single stage gear transmission. The gear meshing keeps clearance of $1 \mathrm{~mm}$, which not only ensures the small friction resistance in the transmission process, but also avoids the skid phenomenon between the rear wheel and the ground.

\subsection{Steering mechanism}

Steering mechanism is the most core part of the whole car design process. Design used disc groove cam as the core of steering mechanism. And the cam structure is simple, compact, convenient design. The desired motion of the follower can be obtained only by designing the proper cam profile. Fig. 3 is the cam steering mechanism:

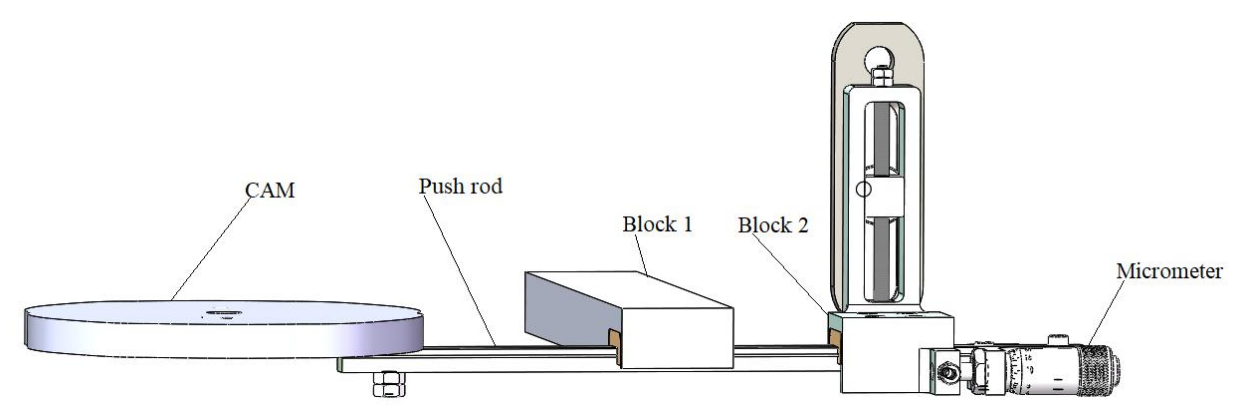

Fig. 3 Steering mechanism design scheme

\section{Inverse method of trajectory}

The design scenario is as follows: Along the track line from distance line $1 \mathrm{~m}$ starting barrier piles is put according to the average spacing of $1 \mathrm{~m}$, an odd number of pile position remains unchanged, the first even number of pile position within plus or minus (200-300) $\mathrm{mm}$ do adjustment (relative to the starting line, comes at a time away from, negative closer), then the even number pile, in turn, as the previous one even adjustment of the same distance from the opposite direction to do, Fig. 4 shows the movement rules of obstacle piles.

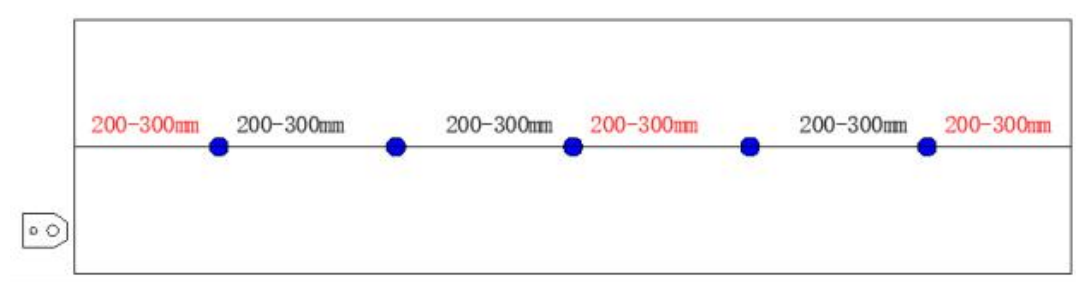

Fig. 4 Pile spacing movement rules 


\subsection{Trajectory and formulation}

Considering the movement of obstacle piles, the car would bypass two long and two short obstacle piles in the process of traveling. Therefore, if the conventional sinusoidal curve is adopted, the error accumulation will be caused. As the traveling distance of the car increases, the possibility of the car body hitting the obstacle piles is greater. Fig. 5 shows that when the pile moves $300 \mathrm{~mm}$, the trolley moves along a cosine curve.

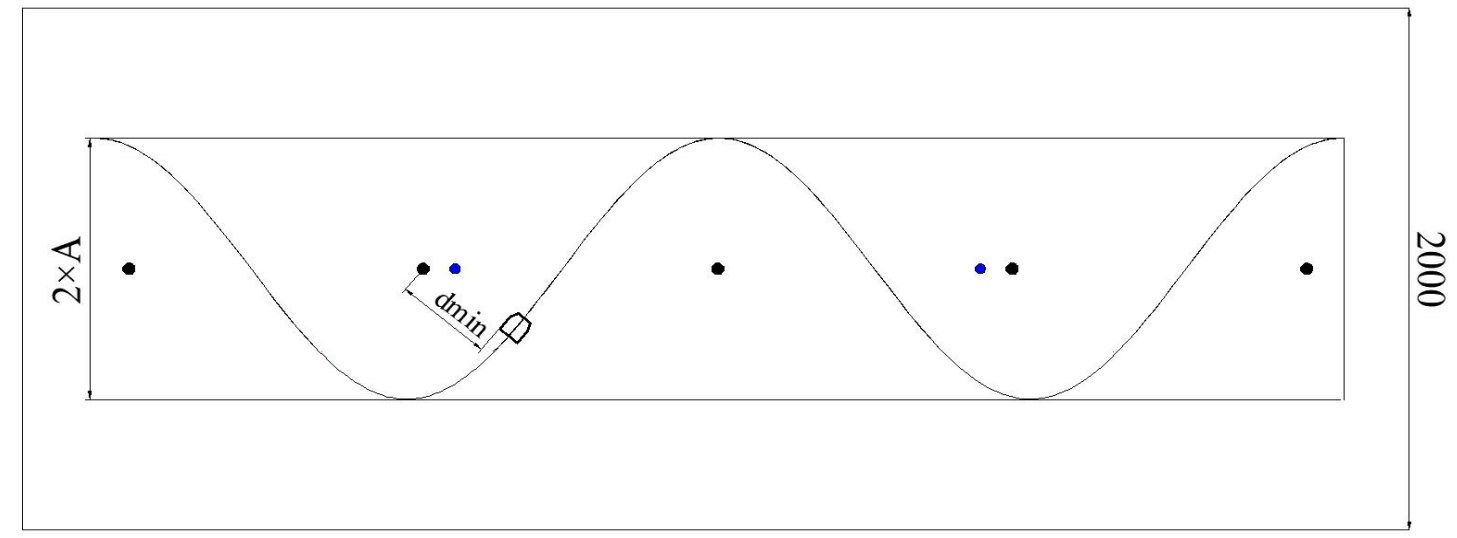

Fig. 5 The trajectory of the trolley

Suppose the trajectory curve equation was:

$$
\mathrm{y}=\operatorname{Acos}(\omega \mathrm{x}+\Phi)
$$

In the formula, $A=355 \mathrm{~mm}, \omega=2 \pi / T, T=2000 \mathrm{~mm}$. known $(x 0, y 0)=(T / 2-300,0)$.

Since the car started from the starting point, the Assume that the point closest to the moving pile coordinate point of the first moving pile was on the curve was $(x, y)$.

$$
\begin{gathered}
f=D_{\min }^{2}=\left(\mathrm{x}_{0}-\mathrm{x}\right)^{2}+\left(\mathrm{y}_{0}-\mathrm{y}\right)^{2} \\
\frac{d f}{d x}=2 x-2 x_{0}-2 y_{0} \frac{d y}{d x}+2 y \frac{d y}{d x}
\end{gathered}
$$

When $\frac{d f}{d x}=0, \quad f$ had a maximum value. At this point, $(x 0, y 0)=(x, y)$, namely, was the point closest to the moving pile. MATLAB was used to obtain $(x 0, y 0)=(644.31,199.74)$. Based on the above conditions, the nearest distance between the obstacle pile and the trolley was obtained:

$$
d_{\text {min }}=\sqrt{\left(\mathrm{x}_{0}-\mathrm{X}_{0}\right)^{2}+\left(\mathrm{y}_{0}-\mathrm{Y}_{0}\right)^{2}}-L / 2-r
$$

In the formula: $L$ was the width of the trolley $160 \mathrm{~mm}$; $r$ was $10 \mathrm{~mm}$.

The solution was $d_{\min }=130.04 \mathrm{~mm}$, and in the same way, $d_{\mathrm{rl}}=172.47 \mathrm{~mm}$.

In the current theoretical state, the distance traveled by the trolley in one cycle was $\mathrm{S} 1$ :

$$
S_{1}=\int_{0}^{T} \sqrt{(\mathrm{dx})^{2}+*(\mathrm{dy})^{2}}=\int_{0}^{T} \sqrt{1+y^{2}} d x
$$

It was Calculated that $\mathrm{S} 1=2522.81 \mathrm{~mm}$.

the trajectory of the trolley was a cosine curve, According to the conventional thinking, when if the moving distance of the moving pile 
reached the maximum, it was very likely for the trolley to bump into the bar, resulting in poor regulation performance of the trolley. In addition, due to the cumulative effect of errors in the process of increasing the travelling distance of the trolley, the phenomenon of continuous bumped into the bar may be caused.

Therefore, considering the safety issues in the process of the trolley circumventing the moving rod, we had drawn up the spline and arc combined trajectory, as shown in Figure 6. Because the spline curve passes through the orderly type value points, and in each type, the first and second derivatives at the value point are continuous, with the characteristics of continuous and uniform curvature change.

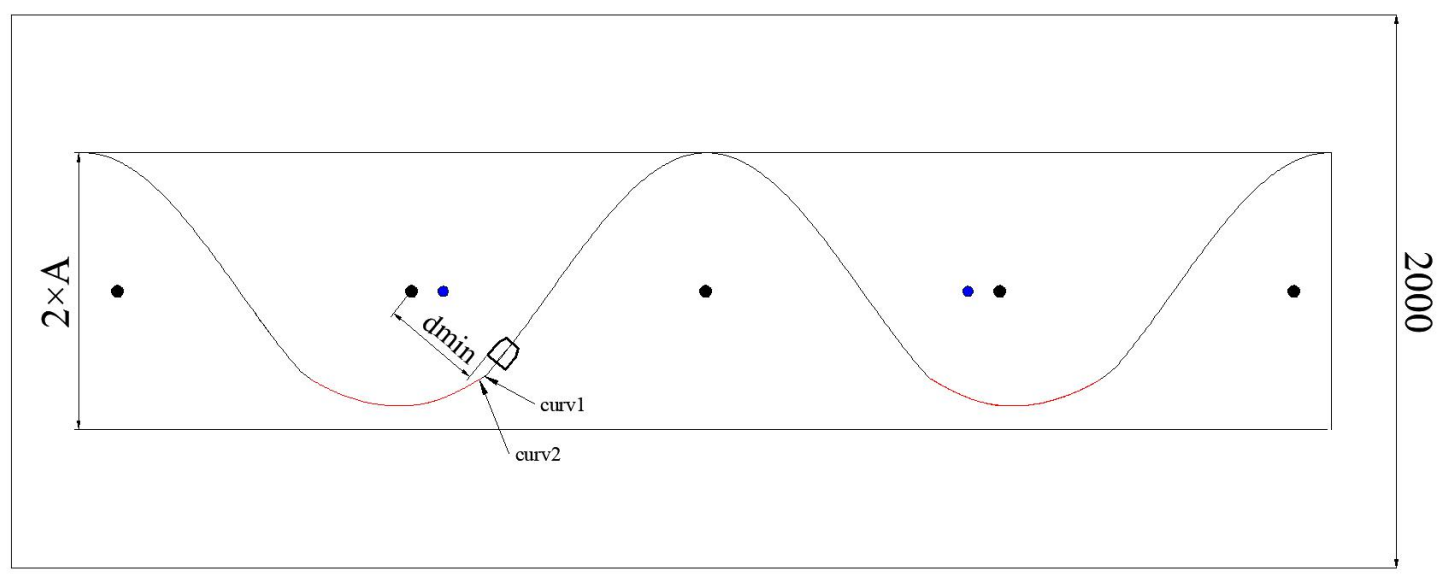

Fig. 6 Combined trajectories of splines and arcs

On the interval $[a, b]=[0,570]$, take $n$ nodes, polation on the spline curve, the interpolation $\mathrm{k}=\mathrm{a}=\mathrm{x} 0<\mathrm{x} 1<\mathrm{x} \ldots<\mathrm{xn}-1=\mathrm{b}$ to perform cubic inter- polynomial was:

$$
y(\mathrm{x})=a_{1} x^{3}+a_{2} x^{2}+a_{3} x+a_{0}
$$

$a_{0} 、 a_{1} 、 a_{2}$ and $a_{3}$ were interpolation coefficients. Since the car body was parallel to

$$
\mathrm{y}(\mathrm{a})=\mathrm{A}, \mathrm{y}(\mathrm{a})^{\prime}=0
$$

the direction when passing the peak of the trajectory curve, the starting point of interpolation would be satisfied:

The center position of the arc segment was (T/2, central Angle was:

$A)$, the radius of the arc was $\mathrm{R}=\mathrm{A}$, and the

$$
\theta=2 \arcsin \left(\frac{T / 2-\mathrm{b}}{R}\right)
$$

At the end of interpolation, the interpolation function should satisfy:

$$
y^{\prime \prime}(\mathrm{b})=1 / \mathrm{R} \quad y(\mathrm{~b})=2 \mathrm{~A}-\mathrm{R} \cos (\theta / 2)
$$

According to the expression of the circular arc, interpolation and the curvature relationship, the the coordinate of the intersection point of the circular arc and the spline curve was (570.00, 142.86). According to the function value of the starting point and the end point of the functional relationship among $a_{0} 、 a_{1} 、 a_{2}$ and $a_{3}$ could be obtained: 


$$
\left(\begin{array}{c}
2 A-R \cos (\theta / 2) \\
\mathrm{A} \\
0 \\
1 / \mathrm{R}
\end{array}\right)=\left(\begin{array}{cccc}
1 & b^{3} & b^{2} & b \\
1 & a^{3} & a^{2} & a \\
0 & 3 a^{3} & 2 a & 1 \\
0 & 6 b & 2 & 0
\end{array}\right) \times\left(\begin{array}{c}
a_{0} \\
a_{1} \\
a_{2} \\
a_{3}
\end{array}\right)
$$

Using MATLAB, the solution was as follows:

$$
\left(\begin{array}{l}
a_{0} \\
a_{1} \\
a_{2} \\
a_{3}
\end{array}\right)=\left(\begin{array}{c}
355.00 \\
1.41 e^{-6} \\
-1.70 e^{-3} \\
0
\end{array}\right)
$$

Then the interpolation polynomial was:

$$
y(\mathrm{x})=1.41 \mathrm{e}^{-6} x^{3}-1.70 e^{-3} x^{2}+355
$$

The data points were uniformly selected on the tween the spline curve and the circular arc was preset spline trajectory. According to the known smooth, the overall curvature changed uniformdata points, combined with the obtained ly, and there was no abrupt change.

difference polynomial, the spline cubic interpo- At the same time, when the spline - arc lation was realized by MATLAB, and the cur- combination curve was used as the car moving vature changes of the car during the half-cycle track, the following could be obtained: trajectory were calculated. The junction be-

$$
\begin{gathered}
\mathrm{d}_{\min 2}=191.14 \mathrm{~mm} \quad d_{\mathrm{r} 2}=178.19 \mathrm{~mm} \\
S_{2}=2\left(\mathrm{~L}_{\text {spline }}+\mathrm{L}_{\text {arc }}\right)=2562.67 \mathrm{~mm}
\end{gathered}
$$

\begin{tabular}{|c|c|c|c|}
\hline Symbol & Meaning & Symbol & Meaning \\
\hline $\mathrm{L} / \mathrm{mm}$ & Car width & $\theta /\left(^{\circ}\right)$ & $\begin{array}{c}\text { Arc segments correspond } \\
\text { to central angles }\end{array}$ \\
\hline $\mathrm{A} / \mathrm{mm}$ & Track amplitude & Curv1/mm-1 & $\begin{array}{c}\text { The radius of curvature of } \\
\text { the arc at the junction }\end{array}$ \\
\hline $\mathrm{dmin} 1 / \mathrm{mm}$ & $\begin{array}{l}\text { Shortest distance from rear } \\
\text { wheel to obstacle stump } \\
\text { (cosine path) }\end{array}$ & Curv2/mm-1 & $\begin{array}{l}\text { The radius of curvature of } \\
\text { the junction spline curve }\end{array}$ \\
\hline $\mathrm{dr} 1 / \mathrm{mm}$ & $\begin{array}{c}\text { Dangerous distance of car } \\
\text { near peak (cosine path) }\end{array}$ & $\mathrm{R} / \mathrm{mm}$ & $\begin{array}{c}\text { Arc segments correspond } \\
\text { to arc radii }\end{array}$ \\
\hline $\mathrm{D} / \mathrm{mm}$ & $\begin{array}{c}\text { Move the pile to the nearest } \\
\text { point of the trajectory }\end{array}$ & $\beta /\left(^{\circ}\right)$ & The front wheel angular \\
\hline $\mathrm{S} 1 / \mathrm{mm}$ & $\begin{array}{c}\text { The length of a sinusoidal } \\
\text { trajectory of a period }\end{array}$ & $r_{0} / \mathrm{mm}$ & Cam base circle radius \\
\hline $\mathrm{r} / \mathrm{mm}$ & Cross section radius of & $\mathrm{l} / \mathrm{mm}$ & Conductor \\
\hline
\end{tabular}

\subsection{Establishment of cam mathematical model}

Table1 Symbolic meaning

AJERR: http://escipub.com/american-journal-of-engineering-research-and-reviews/ 
Xing Li et al,. AJERR, 2021; 4:27

\begin{tabular}{|c|c|c|c|}
\hline & obstacle pile & & \\
\hline $\mathrm{dmin} 2 / \mathrm{mm}$ & $\begin{array}{l}\text { Minimum distance from rear } \\
\text { wheel to obstacle pile (spline - } \\
\text { arc path) }\end{array}$ & $\mathrm{T} / \mathrm{mm}$ & The push rod length \\
\hline $\mathrm{dr} 2 / \mathrm{mm}$ & $\begin{array}{c}\text { Danger distance of car near } \\
\text { peak (spline - arc path) }\end{array}$ & $\varepsilon / \mathrm{mm}$ & $\begin{array}{l}\text { Distance between the front } \\
\text { wheel shaft and the push } \\
\text { rod shaft }\end{array}$ \\
\hline$\sigma / \mathrm{mm}$ & Radius of curvature & $\delta / \mathrm{mm}$ & Cam slip value \\
\hline Lspline & $\begin{array}{l}\text { The length of the spline } \\
\text { segment }\end{array}$ & Larc & $\begin{array}{c}\text { The length of the arc } \\
\text { segment }\end{array}$ \\
\hline$\rho / m m$ & $\begin{array}{l}\text { Curvature radius value of } \\
\text { track points }\end{array}$ & $\rho m a x / m m$ & $\begin{array}{c}\text { Maximum radius of } \\
\text { curvature }\end{array}$ \\
\hline $\mathrm{T} / \mathrm{mm}$ & String of tolerance & $\alpha /\left(^{\circ}\right)$ & Cam Angle per bisection \\
\hline$\xi / \mathrm{mm}$ & Radius of bearing & $\triangle \mathrm{x} / \mathrm{mm}$ & $\begin{array}{c}\text { Distance between actual } \\
\text { profile and envelope }\end{array}$ \\
\hline$\rho \min / \mathrm{mm}$ & Minimum radius of curvature & $\mathrm{j} /(\mathrm{a})$ & Cam equal fraction \\
\hline
\end{tabular}

Fig. 7 is the schematic diagram of the turning front wheel edge was always tangent to the process of the trolley. During the moving track line, so the polar coordinate system as process of the trolley, the tangent line of the shown in the figure was established.

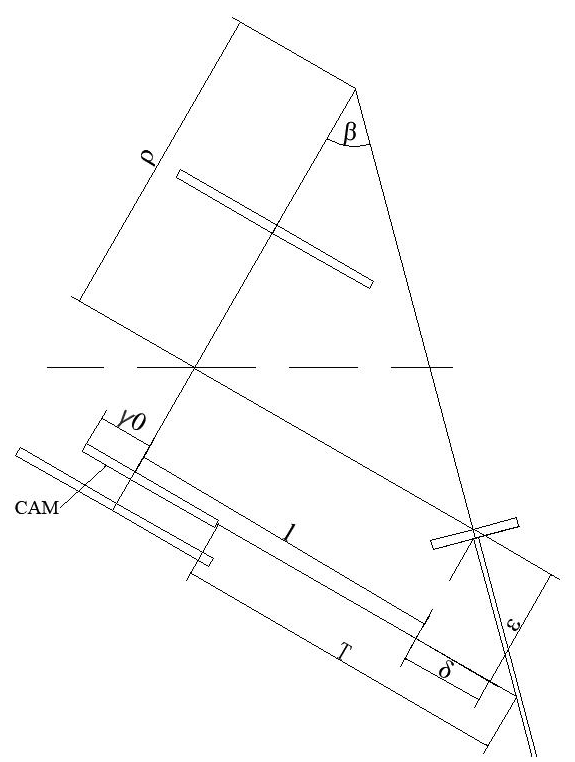

Fig. 7 The schematic diagram of trolley turning

Due to the use of disk-shaped internal grooves geometric parameters could be obtained from against the center cam, the relationship be- the geometric relationship shown in the figure: tween the slide value of the cam and other

$$
\tan (\beta)=l / \rho
$$




$$
\delta=\left\{\begin{array}{l}
\varepsilon \times l / \rho, \beta>0 \\
-\varepsilon \times l / \rho, \beta<0
\end{array}\right.
$$

The slip value of the push rod is only related to the radius of curvature at the point of trajectory change. In drawing according to the motion rule of follower cam profile, with the method of reverse uniform motion Angle, follow the principle of "steep close slow hydrophobic". Because we used the direct reverse path cam, the trajectory curvature changed in the trajectory of large section of cam contour presented a "steep", little change in curvature of track section of cam profile presented a "slow".

The proposed trolley trajectory curve was established in UG software, and $\mathrm{j}(\mathrm{j}=0,1,2, \cdots$, $\mathrm{N})$ data points were established. The variation rule of the one-period curvature radius of the car track is shown in Table2:

Table2 Curvature changes of the trajectory within one week

\begin{tabular}{ccccccc}
\hline State & Set out & Turn right & Turn right over & Turn left & The left end & Turn right \\
\hline$\rho$ & $\rho \operatorname{cmin}$ & $\uparrow$ & $\infty$ & $\infty_{\infty \rightarrow R \rightarrow \infty}$ & $\infty$ & $\downarrow$ \\
\hline
\end{tabular}

In the first cycle of the trajectory, the curvature of the beginning segment and the end segment changed obviously, and here the cam contour showed a "steep" trend, while in the middle segment, it showed a "slow" trend. Therefore, when inserting the point set $\mathrm{J}$, the form of chord tolerance was adopted, and the points were densely distributed in the interval with large curvature changes, while dispersed in the interval with gentle curvature changes. Fig. 8 is the curvature radius distribution of UG extraction.

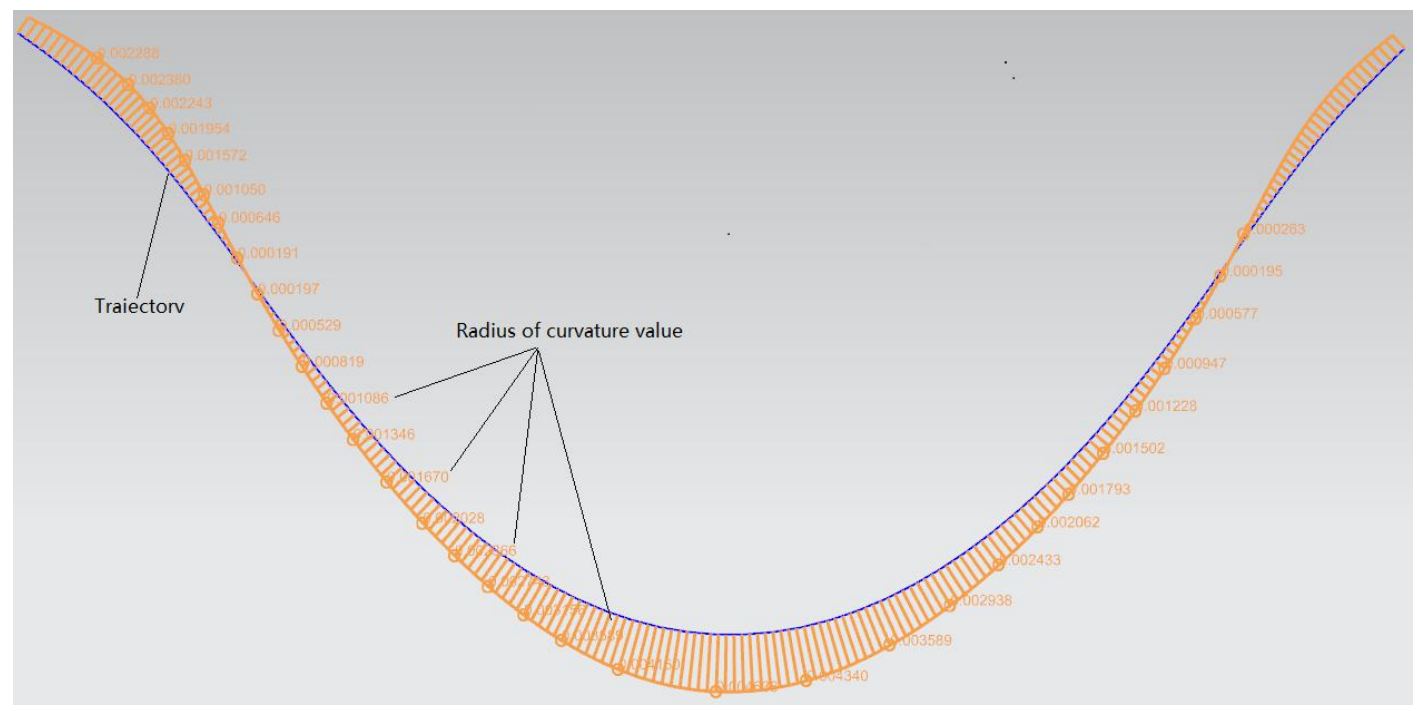

Fig. 8 UG extraction of trajectory curvature radius value

Since the cam rotated once a week and the car traveled a cycle distance, without considering the friction, car assembly clearance and manufacturing error, the front wheel rolled through the above set of points in turn in theory.
For each cam rotation Angle, the front wheel's touching point was corresponding to a unique point on the track. Divide the cam profile into $\mathrm{J}$ parts, and establish the coordinate system as shown in Fig. 9. If the chord tolerance 
$\mathrm{T}=2.54 \mathrm{~mm}$ was set during the arrangement of the point set, the coordinate $(X, Y)$ of the ray with the corresponding Angle $\theta^{\prime}=\mathrm{N} x \alpha$ intersection point between the cam rim and the

$$
P(\mathrm{~N}):\left\{\begin{array}{l}
X=\left(\delta(\mathrm{N})+\gamma_{0}\right) \times \cos (2 \pi \mathrm{N} / \mathrm{j}) \\
Y=\left(\delta(\mathrm{N})+\gamma_{0}\right) \times \sin (2 \pi \mathrm{N} / \mathrm{j})
\end{array}(\mathrm{N}=0,1,2, \cdots, \mathrm{j})\right.
$$

$\mathrm{P}(\mathrm{N})$ was the Nth point of the cam profile coordinate of this point.

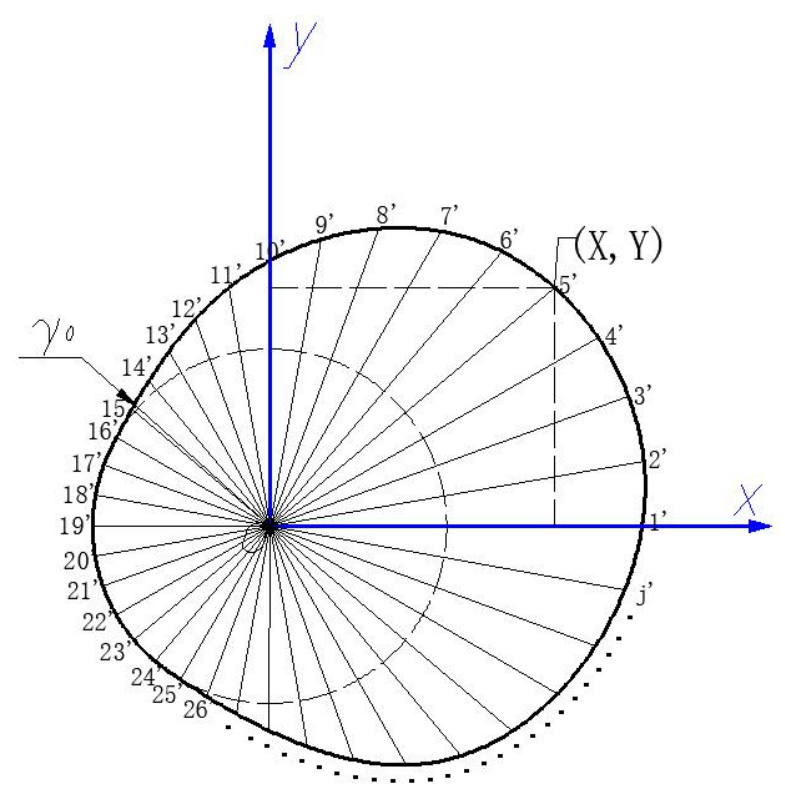

Fig. 9 Coordinate model of cam contour points

If the radius of the base circle $\gamma_{0}=20$, the number of track points set $\mathrm{J}=60$, the vehicle length $L=117.5 \mathrm{~mm}$, the length of the push rod

$$
\delta_{\mathrm{j}}= \pm \varepsilon \times \tan (\arctan (l / \rho))+\rho_{\min }
$$

$\rho_{\min }$ was the smallest value among the $\mathrm{j}$ push values obtained, and this value could be added to shift the push curve above the $X$-axis for easy processing. \pm depended on the $\beta$ value. The coordinate information of each distribution point on the center line of the cam could be obtained according to the curve of the push path of the cam. As a discoid cam with a diagonal groove was adopted, the bearing rolls in the groove and the radius of the outer ring of the bearing was $2.5 \mathrm{~mm}$, the theoretical profile calculated was the track of the center of the
$\mathrm{T}=75 \mathrm{~mm}$, and the distance between the front wheel and the push rod axis $\varepsilon=22 \mathrm{~mm}$, then the push rod push value at the corresponding position of the cam rotation was as follows:

bearing. The distribution of the bearing on the trajectory was simulated by MATLAB programming. It was concluded that the contour calculated by the model was smooth and hadn't dead point, and the bearing center wouldn't appear distortion during the movement of the contour.

\subsection{Cam 3D model establishment}

Import the coordinate point information of the theoretical profile of the cam by using the point function in the file in the UG modeling environment, and connected each point with art splines, as shown in Figure 10. 


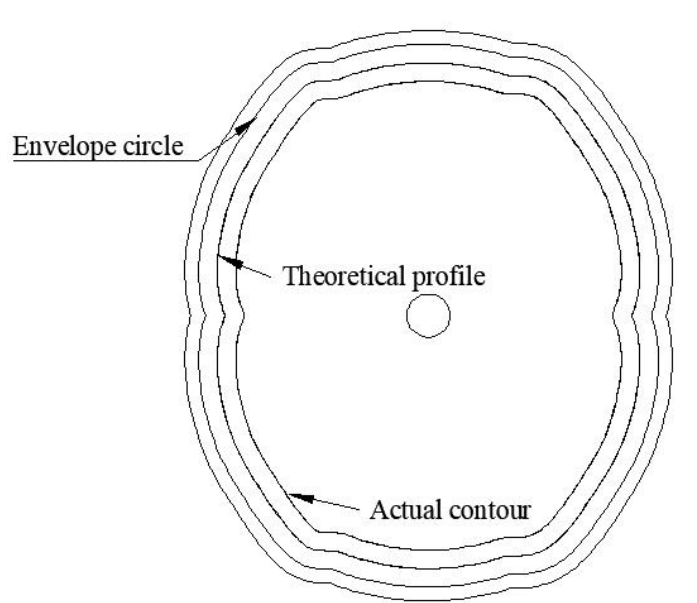

Fig. 10 Cam wireframe diagram

The theoretical contour, actual contour and the envelope were equidistance. The distance be-

$$
\Delta x=2 \xi
$$

According to the analysis of the design software, figure had a minimum value. The minimum the curvature radius at the point shown in the value was:

$$
\rho_{\text {min }}=5.178>\xi
$$

Therefore, the bearing could be rolled smoothly model of the cam was established by using the through all points of the groove, and there stretch command, as shown in Figure 11. would be no sticking phenomenon. The 3D

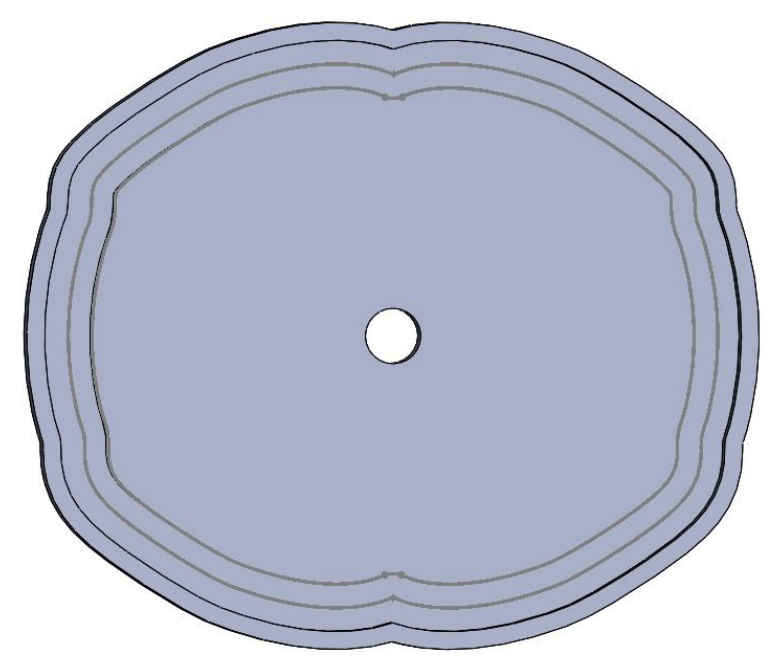

Fig. 11 3D model of cam

\section{Track simulation}

The mathematical model established above was sorted out and some initial parameters were set, the initial value as shown in table 3 . Table 3 symbols and meaning is the same as in Table 1. By using mathematical model builted by the push to get the cam model simulate the trajectory, the theory of simulation trajectory coincidence degree was high, and to formulate Theoretically, it could effectively avoid obstacles. 
Table 3 Initial value Settings

\begin{tabular}{cccccc}
\hline$\gamma_{0} / \mathrm{mm}$ & $\mathrm{l} / \mathrm{mm}$ & $\mathrm{T} / \mathrm{mm}$ & $\varepsilon / \mathrm{mm}$ & $\mathrm{j} /(\mathrm{a})$ & $\mathrm{A} / \mathrm{mm}$ \\
\hline 20 & 117.5 & 75 & 22 & 60 & 355 \\
\hline
\end{tabular}

\section{Entity modeling and validation}

In order to avoid excessive energy loss and ensure smooth start, the mass of the car should be as light as possible. Taking various factors into consideration, aluminum alloy is selected as the main material for the processing of car parts. For the parts with low requirements on precision and strength, such as winding wheel and starter, 3D printing was adopted for processing, which not only saved cost, but also has high processing efficiency. In order to make the weight reduction effect better, the bearing and other parts in the condition of meeting the strength requirements of a series of uniform drilling process holes, so that the weight reduction effect was more obvious. The SolidWorks software was used to design each part of the car, and the model design effect was shown in Fig. 12.

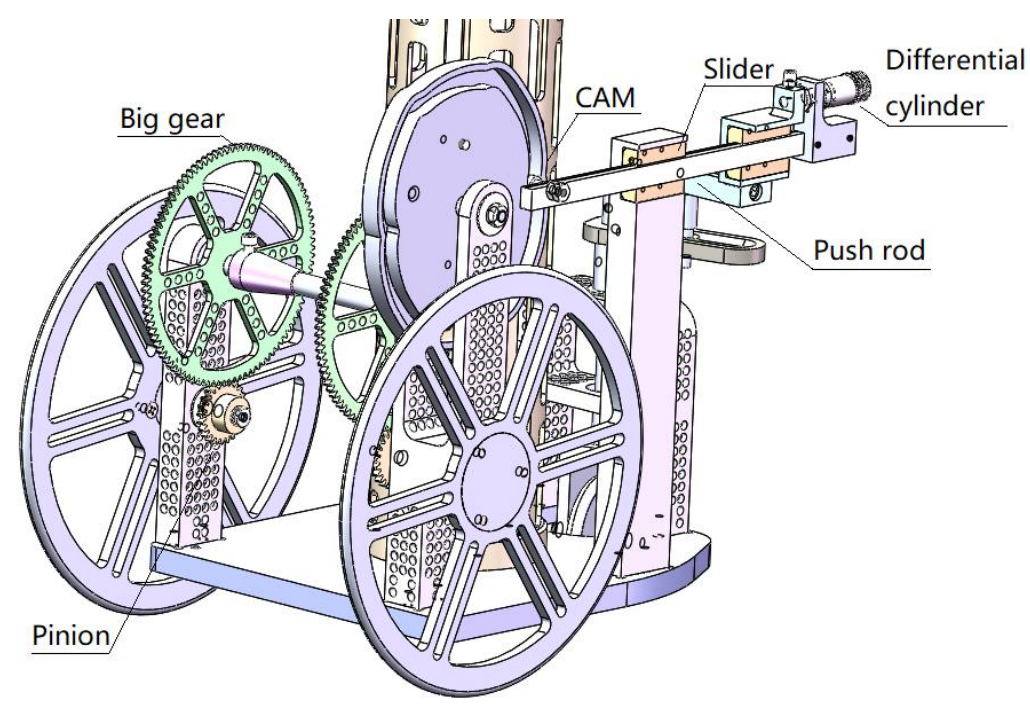

Fig. 12 3D model of trolley

In order to verify the correctness of the established model, CNC machining center milling, turning, drilling and other processes were used to manufacture the designed parts. The friction between the car and the ground and the air was not considered because of machining errors and fitting tolerances in the assembly process. Therefore, after the car assembly was completed, the length of push rod and swing rod were adjusted to eliminate the above random errors and systematic errors. The period and amplitude of the trolley were adjusted by adjusting the length of the swing rod, and the length of the push rod was fine-tuned so that the trajectory of the trolley could always be distributed on a straight line during the long-distance travel. Through constant adjustment, we finally got the actual moving track of the car. The coincidence degree between the actual trajectory and the theoretical trajectory reached more than $90 \%$. When the moving distance of the moving pile reached the maximum distance of $300 \mathrm{~mm}$, the trolley can successfully skirt 36 obstacle piles 
without the phenomenon of bar collision.

\section{Conclusion}

Through MATLAB simulation and physical experiments, the following conclusions could be drawn: To as a car's steering mechanism, cam mechanism used in the process of the establishment of the cam track reverse method to make the car run path flexibility. combination trajectory was compared with the traditional type of sine curve safety, and coefficient is higher. What's more, the car in the process of marching into the possibility of greatly reduced, optimize the trajectory, guarantee the cam no dead point, and the car running smoothly. No stuck dead, sharp turn and other phenomena occurred. The fine-tuning mechanism could reduce the influence of friction on the car, and the car could move in a certain trajectory in the experiment, with a high degree of coincidence between practice and theory.

\section{References}

[1] Lei Chen, Tongyi Liu and Tongle $\mathrm{Xu}$. Kinematics Analysis and Optimization of Carbon-Free Vehicle of "8"-Shaped Trajectory Based on Spatial RSSR Mechanism[J]. Science Research, 2019, $7(1)$

[2] Xiaojie Ai, Zhaohui Wang, Lei Su and Limin Zhao. Design of Gravity Potential Energy Driven Directional Control Carbon-free Vehicle [J]. Machinery Design \& Manufacture, 2016(04): 157-160. (In Chinese)

[3] Jianwei Liu, Yuhan Li and Nianjiong Yang. Innovative Design and Manufacture of "S" Type Carbon-Free Cars[J]. MATEC Web of Conferences, 2017, 95

[4] Chunyang Wang. Innovative Design of S Type Carbon-free Vehicle Transmission Mechanism [J]. South China Agricultural Machinery,2019,50 (19):68-69. (In Chinese)

[5] Lianggui Pu, Guoding Chen and Liyan Wu. Mechanical Design [M].Beijing: Higher Education Press, 2013. (In Chinese) 